\title{
Functional Perfusion Imaging Using Pseudocontinuous Arterial Spin Labeling with Low-Flip-Angle Segmented 3D Spiral Readouts
}

\author{
Jon-Fredrik Nielsen* and Luis Hernandez-Garcia
}

\begin{abstract}
Arterial spin labeling (ASL) provides quantitative and reproducible measurements of regional cerebral blood flow, and is therefore an attractive method for functional MRI. However, most existing ASL functional MRI protocols are based on either two-dimensional (2D) multislice or 3D spin-echo and suffer from very low image signal-to-noise ratio or through-plane blurring. 3D ASL with multishot (segmented) readouts can improve the signal-to-noise ratio efficiency relative to $2 \mathrm{D}$ multislice and does not suffer from $T_{2}$ blurring. However, segmented readouts require lower imaging flip-angles and may increase the susceptibility to temporal signal fluctuations (e.g., due to physiology) relative to 2D multislice. In this article, we characterize the temporal signal-to-noise ratio of a segmented 3D spiral ASL sequence, and investigate the effects of radiofrequency phase cycling scheme and flip-angle schedule on image properties. We show that radiofrequencyspoiling is essential in segmented $3 D$ spiral ASL, and that $3 D$ ASL can improve temporal signal-to-noise ratio 2-fold relative to 2D multislice when using a simple polynomial (cubic) flip-angle schedule. Functional MRI results using the proposed optimized segmented 3D spiral ASL protocol show excellent activation in the visual cortex. Magn Reson Med 69:382-390, 2013. () 2012 Wiley Periodicals, Inc.
\end{abstract}

Key words: arterial spin labeling; 3D arterial spin labeling; radiofrequency-spoiling; $2 \mathrm{D}$ versus $3 \mathrm{D}$

\section{INTRODUCTION}

Arterial spin labeling (ASL) $(1,2)$ provides quantitative measurements of regional cerebral blood flow in units of $\mathrm{mL} / \mathrm{min}$ per $100 \mathrm{~g}$ of tissue and produces functional activation signals that are well localized to brain parenchyma rather than the veins. ASL techniques are also attractive because they are reproducible across scan sessions (3-5) and across scanners (4). The quantitative and reproducible nature of ASL makes it ideal for low task-frequency functional magnetic resonance imaging (fMRI) studies extending across multiple scan sessions (6) and as a potential biomarker for assessing the functional response to treatment (7-11).

Unfortunately, ASL has inherently low signal-to-noise ratio (SNR) efficiency, due mainly to the relatively small amount of labeled spins in the tissue, but also in part to the

Department of Biomedical Engineering, University of Michigan, Ann Arbor, Michigan, USA

${ }^{\star}$ Correspondence to: Jon-Fredrik Nielsen, Ph.D., 1067 B.I.R.B., 2360 Bonisteel Ave, Ann Arbor, Ml 48109 - 2108. E-mail: jfnielse@umich.edu

Received 24 October 2011; revised 23 February 2012; accepted 24 February 2012.

DOI 10.1002/mrm.24261

Published online 4 April 2012 in Wiley Online Library (wileyonlinelibrary. com).

(C) 2012 Wiley Periodicals, Inc. use of two-dimensional (2D) multislice readouts (12-14). Conventional ASL fMRI measurements are therefore inherently unreliable and require aggressive temporal averaging and long scan times. Furthermore, 2D multislice readouts introduce a slice-dependent bias in blood perfusion quantification, which may affect the extent of "active" brain regions observed in fMRI studies.

Acquisition schemes using 3D k-space trajectories can offer some relief to these problems and have recently motivated development in this area (15-20). More specifically, the SNR efficiency of 3D acquisitions can be greater than that of 2D multislice. Second, 3D ensures the same acquisition delay for the entire imaging volume relative to the labeling period, as the whole volume is excited simultaneously by each radiofrequency (RF) excitation pulse.

Despite the apparent benefits of 3D ASL for functional imaging, several questions remain about the details of the pulse sequence and about its dynamic imaging performance. First, multishot 3D readouts require the use of low flip-angles that reduce the signal, and it is not clear whether segmented 3D ASL holds any SNR benefit over $2 \mathrm{D}$ multislice, and whether the temporal SNR (tSNR) of the ASL signal scales with imaging flip-angle. Note that the pulse sequence discussed in this article is distinct from ASL sequences based on fast spin-echo readouts $(15,16,21)$. Second, to our knowledge, the effect of RF phase cycling scheme on the temporal stability of the segmented 3D ASL signal has not been investigated. Third, the impact of different flip-angle schedules on blurring in the tag-control difference images has also not been investigated. In Ref. 18, the authors used a flip-angle schedule based on the Ernst formula that produces a constant blood signal in the individual tag or control images, but it is not clear whether a simpler functional form that is easier to implement such as a low-order polynomial can offer improved performance in the ASL difference images.

The goal of this article is to investigate the dynamic imaging properties and challenges of a pseudo-continuous ASL (pCASL) imaging protocol based on 3D stack-ofspirals readouts and arrive at a set of recommendations. We use spiral readouts because they are optimally time efficient, such that the total readout duration after each labeling period is minimized. Spirals are also relatively robust to flow and motion inconsistencies between spiral data segments due to inherent oversampling at low spatial frequencies, and are therefore expected to exhibit lower temporal variance compared with rectilinear echoplanar imaging (22). We (i) perform matched 2D and 3D ASL experiments to establish whether segmented 3D spiral offers significant tSNR benefits over 2D multislice, (ii) 


$\left.\begin{array}{l}\text { FIG. 1. Pulse sequence timing diagram for seg- } \\ \text { mented 3D spiral ASL. After a labeling period } \\ \begin{array}{l}\text { and postinversion delay, a "snapshot" of the } \\ \text { magnetization is acquired with a centric stack- } \\ \text { of-spirals 3D echo-planar imaging readout. }\end{array}\end{array}\right) \begin{gathered}\text { PCASL } \\ \text { labeling }\end{gathered}$

examine experimentally the influence of RF phase cycling scheme on tSNR in dynamic spiral segmented 3D ASL, (iii) investigate theoretically the effect of different polynomial flip-angle schedules (constant, linear, quadratic, and cubic) on the kz-space sampling profile, and (iv) apply the proposed segmented 3D spiral ASL protocol to dynamic functional imaging during a visual task. Our measurements indicate that segmented 3D spiral ASL with a starting flip-angle of $15^{\circ}$ achieves a roughly 2 -fold tSNR increase relative to $2 \mathrm{D}$ multislice, in rough agreement with a previous report (20). Our results also show that RF spoiling increases tSNR by $60 \%$ compared to the case of gradientspoiling (constant RF phase), in qualitative agreement with steady-state signal theory. We conclude that RF-spoiled segmented 3D spiral ASL with a cubic flip-angle schedule starting at $15^{\circ}$ can produce high-quality functional activation maps with only modest nominal blurring.

\section{THEORY}

Figure 1 shows the 3D ASL pulse sequence used in this study, which we will refer to as "segmented 3D spiral ASL". The stack-of-spirals readout consists of a train of low-flip-angle excitations $\alpha$, with one $z$ phase-encode being acquired after each excitation. A 2D spiral covering the full $\mathrm{k} x-\mathrm{k} y$ space is sampled after every RF excitation, such that the total number of RF excitations required to sample the full 3D k-space volume equals the number of $\mathrm{kz}$-encoding steps $N$.

\section{$2 \mathrm{D}$ versus $3 \mathrm{D}$}

In steady-state imaging, the theoretical SNR increase of 3D over 2D acquisitions for matched flip-angles is $\sqrt{N}$, where $N$ is the number of $\mathrm{kz}$-encodes. The SNR advantage of 3D can be viewed as a signal averaging effect: each voxel is excited $N$ times (one for each $\mathrm{kz}$-encode), and the noise is therefore reduced by a factor of $1 / \sqrt{N}$. If we assume that the longitudinal magnetization existing prior to each low-flipangle excitation is constant, then we can view segmented 3D ASL imaging as being in a quasi-steady-state, and we therefore believe that the general SNR considerations from steady-state imaging apply here.

However, to mitigate $\mathrm{kz}$-space filtering effects (described below) in segmented 3D imaging, it is necessary to reduce the imaging flip-angle $\alpha$, which reduces the SNR advantage of $3 \mathrm{D}$ by a factor $\sin (\alpha)$. Here, we are assuming centerout $\mathrm{k} z$-space encoding with minimal $\mathrm{k} z$-space filtering effects. The SNR losses are partially mitigated by the fact that the tag-acquisition delay in 2D multislice imaging is slice dependent, as each $2 \mathrm{D}$ slice is excited only once per labeling period using a $90^{\circ}$ excitation pulse ("single-shot" imaging), and hence the tag-acquisition delay for a particular slice is given by the delay between the tag and the time point when the $90^{\circ} \mathrm{RF}$ pulse is applied to that slice. The expected image SNR advantage therefore has a lower bound given by

$$
\frac{\mathrm{SNR}_{3 \mathrm{D}}}{\mathrm{SNR}_{2 \mathrm{D}}}>=\sin (\alpha(1)) \times \sqrt{N}
$$

where $\alpha(n)$ denotes the flip-angle of the $n$th RF excitation. Equation [1] is true for both the ASL (subtraction) image and the SNR of the individual control or tag images. Note that as the $3 \mathrm{D}$ acquisition uses center-out kz-ordering, the ASL signal should be determined primarily by the signal after the first excitation pulse, i.e., it should be proportional to $\sin (\alpha(1))$. However, we must also consider the tSNR,

$$
\mathrm{tSNR} \equiv \frac{\mathrm{SNR}}{\sigma_{\mathrm{t}}}
$$

where $\sigma_{t}^{2}$ is the temporal variance of the ASL signal. It is possible that the temporal variance of $3 \mathrm{D}$ acquisitions is higher than for 2D multislice, e.g., due to image artifacts (e.g., aliasing) caused by shot-to-shot signal inconsistencies caused by physiological signal variations, which would reduce the tSNR benefit of $3 \mathrm{D}$ versus $2 \mathrm{D}$ to a value below that in Eq. [1]. In this article, we measure the tSNR of both 2D multislice and segmented 3D experimentally.

\section{RF Phase Cycling}

We hypothesize that RF-spoiled imaging has reduced sensitivity to flow variations (i.e., cardiac pulsatility) during the readout, and hence exhibits reduced temporal variance compared to non-RF-spoiled acquisitions. This hypothesis is motivated by steady-state signal theory for sequences with very short sequence repetition time (pulse repetition time $\ll T_{1}, T_{2}$ ) (23). In such sequences, the RF phase cycling scheme has a large influence on the acquired signal, and on the sensitivity to blood flow and motion. This is because in the steady-state, non-RF-spoiled gradient-echo sequences contain a superposition of multiple dephased spin ensembles within each voxel (23). A spin moving through one or more voxels will not be in a stable steady-state, which leads to shot-to-shot data inconsistencies and image artifacts. 
To mitigate such flow and motion artifacts, one can use "RF-spoiling," which refers to the use of a linear RF phase increment from shot-to-shot (24). A linear phase increment of $n \times 117^{\circ}$ is commonly used, where $n$ refers to the RF shot number. This RF cycling scheme has the effect that any transverse magnetization existing prior to each RF shot is effectively removed. In RF-spoiled sequences, the spin history from previous pulse repetition times is "erased," and no shot-to-shot transverse spin coherences exist. As a result, RF-spoiled imaging may be more robust to flow variations between shots in segmented 3D ASL.

In the proposed segmented 3D spiral ASL sequence, the signal is not acquired in a steady-state but rather during a transient phase. The transient signal evolution in non-RF-spoiled sequences is quite complex and exhibits oscillations that depend strongly on voxel position. RF spoiling is known to be beneficial in the transient phase as well, and the transient signal has been shown to be well approximated by the Ernst formula, i.e., to be predominantly $T_{1}$ weighted. This indicates that RF spoiling is effective at removing past spin history in transient as well as steady-state imaging. For these reasons, we hypothesize that RF-spoiling reduces the temporal variance of the ASL signal.

\section{kz-Space Filtering}

During the acquisition of the ASL 3D spiral images, after a long period of time dedicated to labeling the arterial blood (approximately $3.5 \mathrm{~s}$ ), the main magnetization vector is tipped toward the $x y$ plane once per $\mathrm{k} z$ encoding step in rapid succession (approximately every 20-30 ms each). Thus, the longitudinal magnetization vector prior to the first RF excitation is almost fully relaxed for the first kz-encoding step but partly saturated at all the subsequent ones. This results in a weighting of the k-space data along the $\mathrm{kz}$-dimension that depends on the flip-angle, $T_{1}$ relaxation rate, and acquisition order. This weighting has a filtering effect on the image along the $z$-dimension.

One possible solution to this problem is to reduce the flip-angle throughout the whole acquisition such that the magnetization vector is equally weighted across all of $\mathrm{kz}$, but this approach reduces the available SNR over all of k-space. Alternatively, increasing the flip-angle gradually, as the available longitudinal magnetization is "spent," can be a more efficient way to use the available magnetization while reducing the weighting along $\mathrm{kz}$.

A second source of $\mathrm{kz}$ filtering arises from the kinetics of the labeled spins in the tissue. Although, ideally, one would like to sample all of $\mathrm{kz}$ when the concentration of tagged spins in the tissue is at equilibrium, in practice, the image is typically acquired while the tag is either buildingup or washing out from the tissue. This is illustrated in Fig. 2, which indicates the timing of the acquisition relative to the kinetics of the tagged spins. In a 2D ASL acquisition, this can manifest itself as a difference in signal intensity between the first and last slice acquired that must be accounted for during quantification as part of the postinversion delay (25). In 3D acquisitions, it results in a second weighting kernel of the data along the $\mathrm{kz}$-dimension.

In this article, we present the results of numerical Bloch calculations of the blurring kernels (in the subtraction

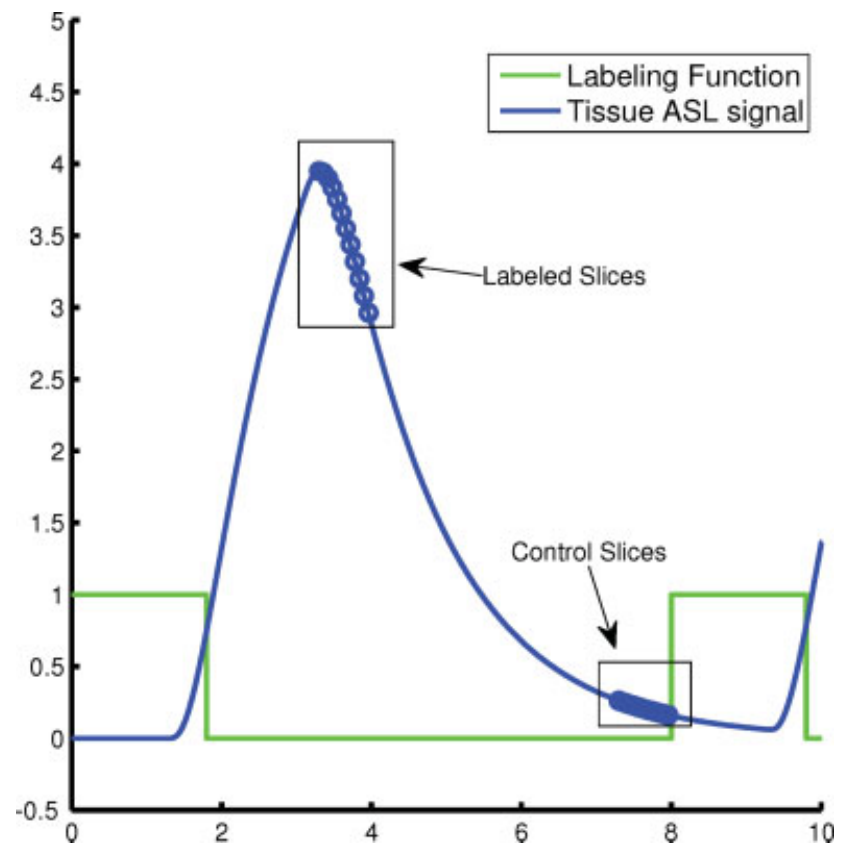

FIG. 2. Build-up and clearance of the inversion tag from the tissue. The labeling function is also plotted for reference and the time of acquisition of the labeled and control images are also indicated. [Color figure can be viewed in the online issue, which is available at wileyonlinelibrary.com.]

images) resulting from either relaxation or inflow effects, to inform the choice of acquisition parameters.

\section{METHODS}

\section{Simulations of kz-Space Filtering}

To estimate the blurring effect on the ASL subtraction image, we simulated the transverse magnetization during a segmented 3D acquisition consisting of a train of 24 lowflip-angle RF pulses ( $24 \mathrm{kz}$-steps). We assumed $T_{1}$ to be $1400 \mathrm{~ms}$ (gray matter at $3 \mathrm{~T}$ ) and that $\mathrm{kz}$ was sampled from the center out. We assumed that each $\mathrm{kz}$ "pancake" was collected within $30 \mathrm{~ms}$. We also assumed that $5 \%$ of the spins in the tissue is replaced by the labeled spins and that the efficiency of the label had decayed at a $T_{1}$ relaxation rate of $1.6 \mathrm{~s}$ during a transit time of $1.2 \mathrm{~s}$. We restricted the possible flip-angle schedules to simple polynomials, as they are easily implemented on the scanner and provide a general and convenient parametrization. We varied the flip-angle according to each of the following schedules: (1) constant flip-angle for all $\mathrm{kz}$ steps, (2) linearly increasing flip-angle between an initial flip-angle $\alpha(1)$ at $\mathrm{kz}=0^{\circ}$ and $90^{\circ}$ at $\mathrm{k} z=\mathrm{k} z \max$, (3) quadratically increasing from $\alpha(1)$ to $90^{\circ}$, and (4) cubically increasing from $\alpha(1)$ to $90^{\circ}$. For each case, we varied the initial flip-angle $\alpha(1)$ from $10^{\circ}$ to $30^{\circ}$. We then calculated the transverse magnetization at the beginning of each $\mathrm{kz}$ shot to determine the observed signal in the control and in the labeled image separately. We obtained the blurring kernel along the $z$ direction of the perfusion-weighted image by subtracting the labeled from the control data.

We then investigated the blurring kernel produced by inflow effects, independently of the $T_{1}$ and flip-angle 
Table 1

Summary of acquisitions comparing 2D and 3D ASL (volunteer studies I).

\begin{tabular}{lccc}
\hline $\begin{array}{l}\text { Acquisition } \\
\text { type }\end{array}$ & $\alpha$ schedule & $\begin{array}{c}\text { Starting } \\
\text { flip-angle }(\alpha(1))\end{array}$ & $\begin{array}{c}\text { RF phase } \\
\text { cycling }\end{array}$ \\
\hline 2D multislice & N/A & $90^{\circ}$ & N/A \\
3D & As in Ref. 18 & $8^{\circ}$ & RF-spoiled \\
3D & Cubic & $8^{\circ}$ & RF-spoiled \\
3D & Cubic & $15^{\circ}$ & RF-spoiled \\
\hline
\end{tabular}

effects just discussed (i.e., we did not consider the effects of image acquisition on the label). The kinetics of the tag buildup and clearance were simulated using a two compartment convolution model, as described previously (26). Perfusion was assumed to be $0.01 \mathrm{~mL} / \mathrm{s} / \mathrm{g}$ and transit time was varied from 1.1 to $2.3 \mathrm{~s}$. We assumed that the arterial contribution to the ASL signal was suppressed and only the microvascular and parenchymal contributions were considered. The ASL sequence timing parameters were assumed to be: pulse repetition time $=4 \mathrm{~s}$, labeling duration $=2 \mathrm{~s}$, and postinversion delay $=1.5 \mathrm{~s}$. Each kz plane was assumed to be collected in $20 \mathrm{~ms}$, beginning at the center of k-space (center-out view ordering).

\section{Volunteer Studies I: Baseline ASL Measurements}

All human volunteer experiments were performed on a GE Signa 3T scanner using the vendor-provided quadrature birdcage transmit-receive headcoil, and in compliance with our institutional IRB. The inversion tagging duration was $1800 \mathrm{~ms}$ and the postinversion delay was $1500 \mathrm{~ms}$, leaving $700 \mathrm{~ms}$ for acquisition of the k-space data ( $30 \mathrm{~ms}$ per plane). Prior to the ASL experiment, the pseudocontinuous pulses were optimized to compensate for off-resonance effects as in Ref. 27. 3D pseudocontinuous ASL gradient echo spiral images were obtained with pulse repetition time $/$ echo time $=4000 / 4 \mathrm{~ms}$, flip-angle $=$ $8^{\circ}$ or $15^{\circ}$, field of view $=24 \mathrm{~cm}$, slab thickness $=11 \mathrm{~cm}$, and matrix size $=64 \times 64 \times 22$. Planes along the $\mathrm{kz}$ dimension were sampled starting at the center of k-space and moving outward alternating between negative and positive $\mathrm{k} z$.

In all studies, images were reconstructed from the $3 \mathrm{D}$ k-space data by first regridding the $2 \mathrm{D}$ spiral data at each plane and performing a 2D Fourier transform at each plane. The gridding was done using the spiral k-space trajectory that was input to the scanner, and we did not measure the actual trajectory experimentally. A second Fourier transform was then performed along the $z$-dimension. The reconstructed images were pairwise-subtracted, to obtain a perfusion-weighted time series.

For the 2D acquisitions, axial slices were acquired sequentially from the most inferior to the most superior slice. Each slice was acquired only once per tagging period $(\mathrm{NEX}=1)$.

Two sets of volunteer studies were performed. Volunteer studies I was designed to compare the performance of 2D multislice and 3D ASL and to investigate the effect of RF phase cycling on the tSNR of the baseline ASL signal. The temporal mean and standard deviation were computed at each pixel of the subtracted images to obtain measures of the tSNR of the perfusion-weighted time series. Five subjects were imaged in volunteer studies I.
For the 2D versus 3D comparison, four ASL acquisitions were performed in each subject: one 2D multislice and three different 3D acquisitions (Table 1). This was done to investigate the effect of flip-angle on signal strength and temporal variance and to compare the tSNR of a cubic flipangle schedule with the schedule proposed in Ref. 18. Only the 10 central slices were used in the quantitative analysis, to avoid the frontal sinus and ear canals (which suffer from severe signal loss and distortion) and to avoid signal contamination from wrap-around along $z$.

To investigate RF phase cycling effects, another set of three ASL acquisitions were acquired in each subject: 3D ASL acquisitions with constant and random phase cycling and RF-spoiling (Table 2). Random and RF-spoiling schemes were chosen to investigate whether the signal variance is sensitive to the precise details of the RF spoiling scheme. In particular, random phase cycling should be less efficient than RF spoiling (24). RF-spoiling was implemented with a linear phase increment of $n \times 117^{\circ}$ as described above. Pseudorandom phase cycling was implemented using a random number generator from Ref. 28.

\section{Volunteer Studies II: Visual fMRI Experiments}

In the second set of volunteer studies, three subjects were scanned using the proposed 3D spiral pseudocontinuous ASL acquisition scheme while undergoing a visual stimulation paradigm. The image protocol was the same as in volunteer studies I and used a starting flip-angle of $15^{\circ}$, a cubic schedule, and RF-spoiling. The subjects were stimulated visually with a circular checkerboard pattern flashing at $8 \mathrm{~Hz}$ for $30 \mathrm{~s}$ followed by $30 \mathrm{~s}$ of no stimulation. This stimulation cycle was repeated five times.

The data were reconstructed and surround subtracted to obtain a time series of perfusion-weighted images. Statistical analysis was carried out using FASL01 (http://fmri.research.umich.edu/resources/software/shared _code.php). A general linear model of the activationinduced perfusion time course was constructed by convolution of a canonical hemodynamic response function with a box-car function reflecting the stimulation paradigm. The time course at each pixel was fitted with this model using least-squares. The fit yielded estimates of the signal change due to the visual stimulation paradigm as well as the variance of those estimates. A map of the $Z$ statistic at each pixel was computed from the parameter estimates and their variance.

\section{RESULTS}

\section{k-Space Filtering}

Figure 3 shows the magnitude of the subtraction of the control and labeled image's transverse magnetization vector at

Table 2

Summary of acquisitions used to measure effect of different RF phase cycling schemes (volunteer studies I).

\begin{tabular}{lccl}
\hline $\begin{array}{l}\text { Acquisition } \\
\text { type }\end{array}$ & $\alpha$ schedule & $\begin{array}{c}\text { Starting } \\
\text { flip-angle }(\alpha(1))\end{array}$ & $\begin{array}{c}\text { RF phase } \\
\text { cycling }\end{array}$ \\
\hline 3D & Cubic & $15^{\circ}$ & Constant \\
3D & Cubic & $15^{\circ}$ & Random \\
3D & Cubic & $15^{\circ}$ & RF-spoiled \\
\hline
\end{tabular}




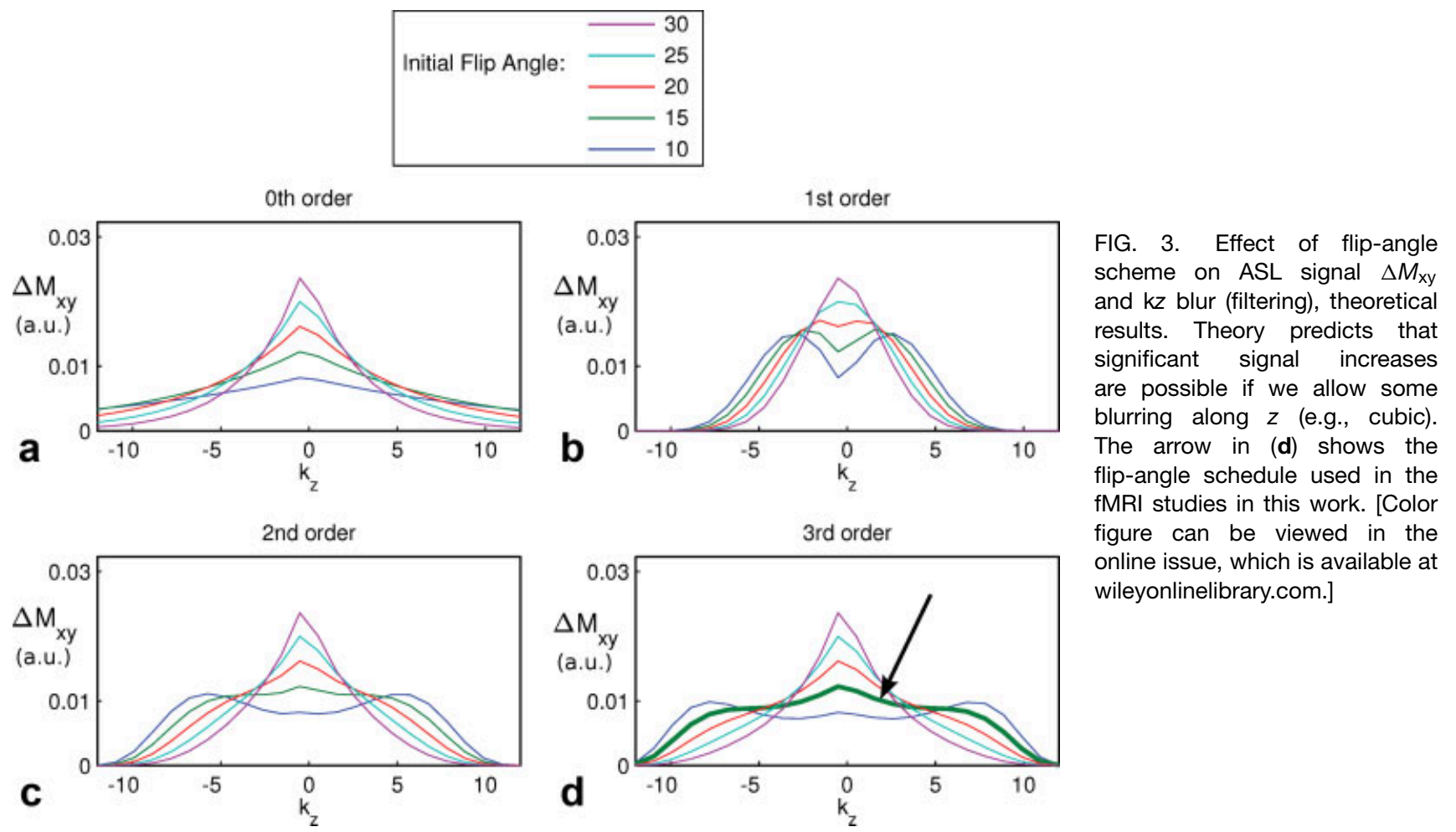

the beginning of the acquisition of each kz-step. This is effectively the same as the blurring kernel (or point-spread function in the $z$-direction) of the perfusion image obtained for each flip-angle schedule. As the figure indicates, the only way to obtain a flat profile is to maintain very low constant flip-angles, but this is done at a great cost of SNR.

Figure 4 shows the normalized blurring kernel along the $z$-axis resulting from the clearance of the label during acquisition of the 3D k-space slab for transit times ranging from 1.1 through $2.3 \mathrm{~s}$. This blurring kernel is fairly mild, as no frequencies are suppressed more than $40 \%$. We note that when the transit time is less than the postinversion delay $(1.5 \mathrm{~s})$, as is common in practice, the kernel acts as a low pass filter. When the transit time is longer than the postinversion delay, on the other hand, the kernels behavior is that of a high pass filter.
FIG. 4. Inflow blurring filter. [Color figure can be viewed in the online issue, which is available at wileyonlinelibrary.com.]

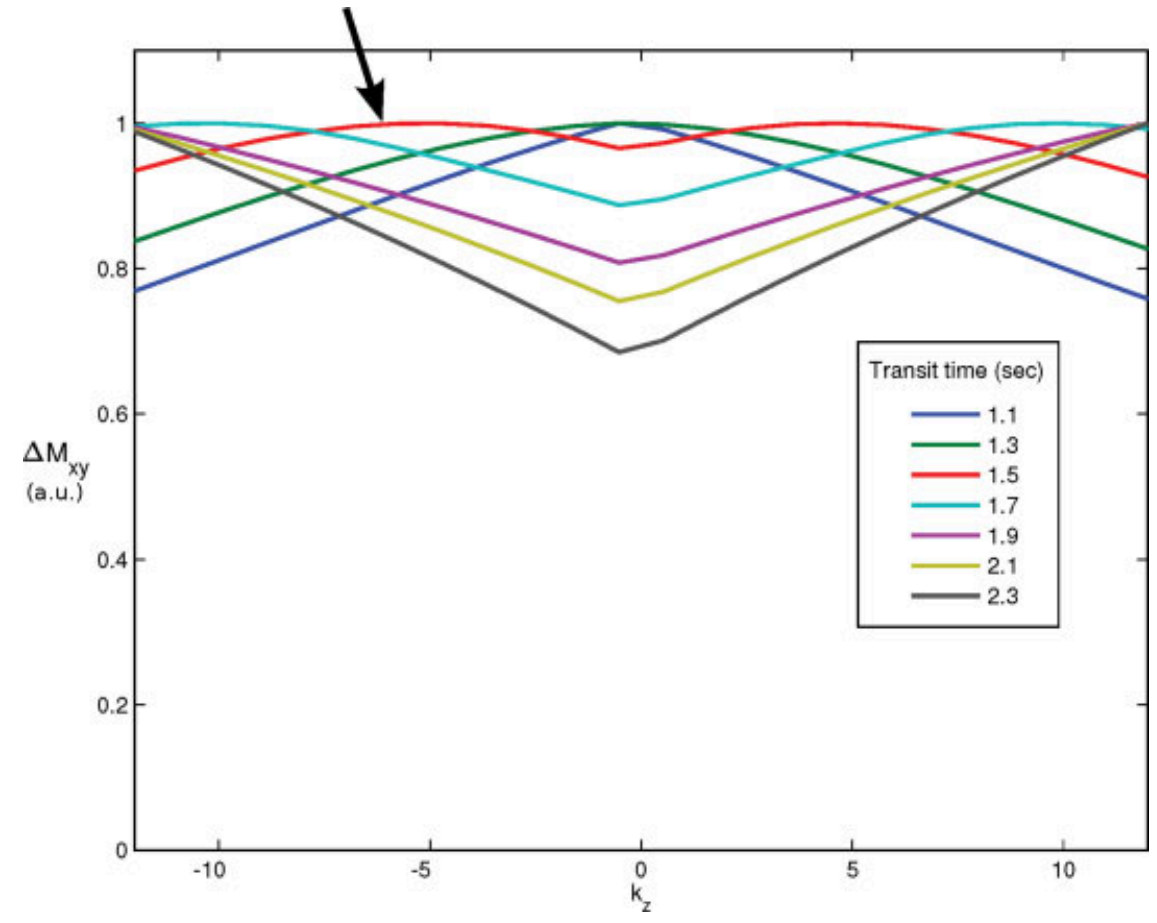




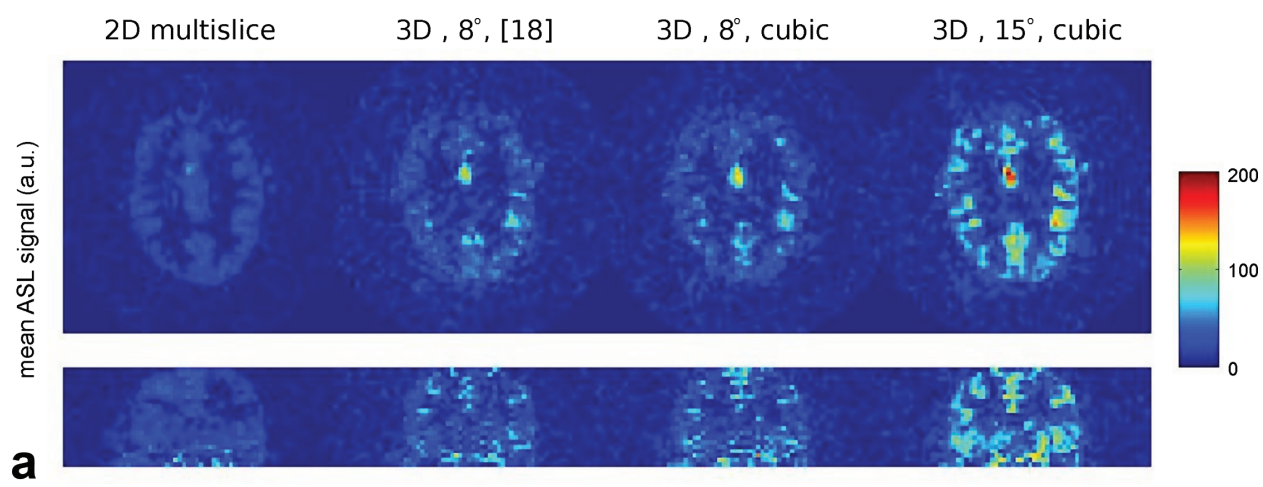

FIG. 5. tSNR comparison of $2 \mathrm{D}$ and 3D ASL (subject 1). Results are shown for 2D multislice and for three different 3D acquisitions. Both axial and coronal views are shown. 3D imaging with $15^{\circ}$ flipangle (right) produces significantly higher ASL signal (a) and only moderately higher temporal variance (b) compared to $2 \mathrm{D}$ multislice.
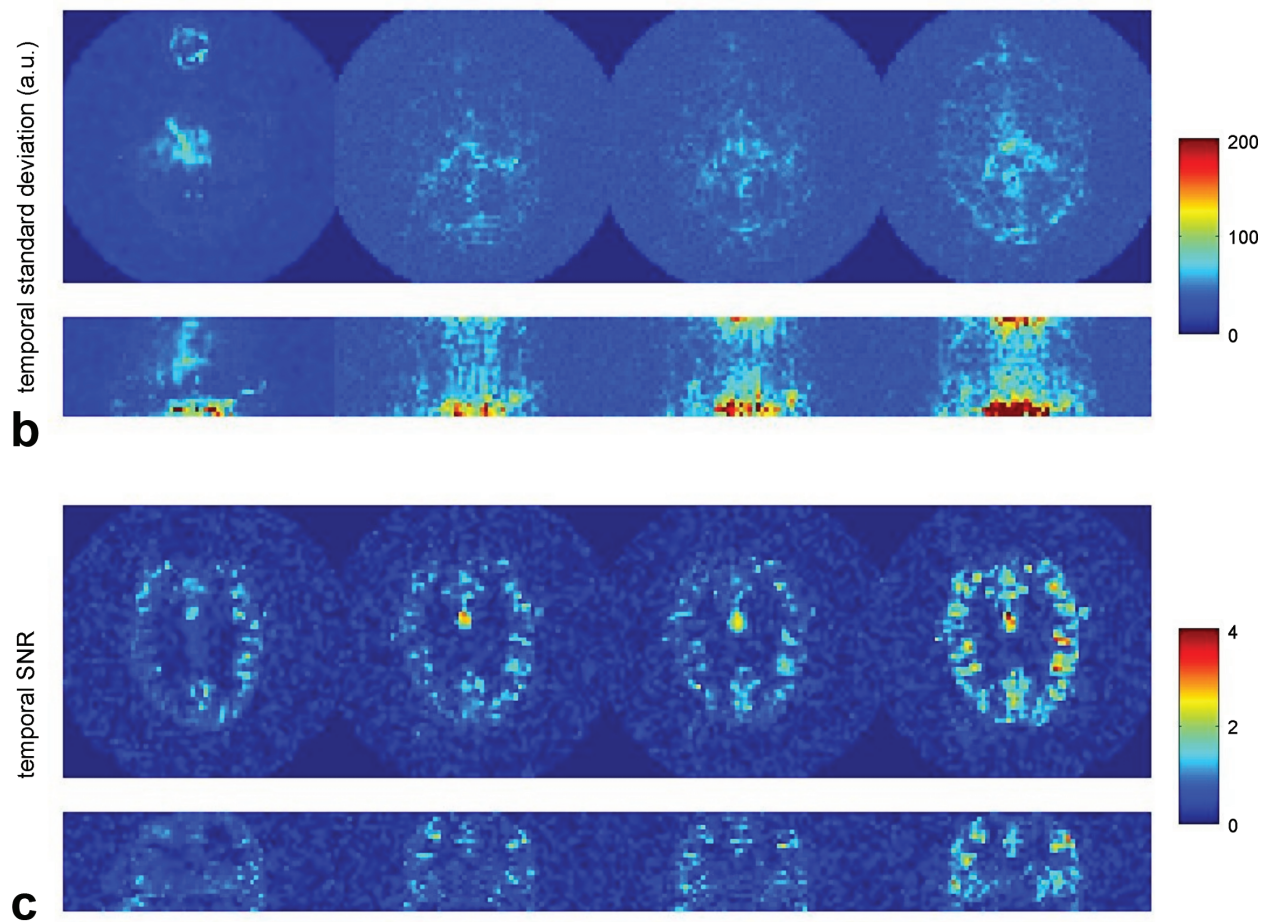

\section{$2 \mathrm{D}$ versus $3 \mathrm{D}$}

Figure 5 shows representative images comparing 2D and 3D in one subject. We can make several observations from these images: first, the $3 \mathrm{D}$ signal for $15^{\circ}$ flip-angle is significantly higher than 2D multislice, and the 3D signal for $8^{\circ}$ flip-angle is similar to 2D multislice. Second, the temporal standard deviation $\left(\sigma_{t}\right)$ of $3 \mathrm{D}$ is higher than for $2 \mathrm{D}$, as anticipated from the theory discussion above. Third, $\sigma_{t}$ is not strongly influenced by flip-angle. Fourth, the Gai (18) and cubic flip-angle schedules produce similar ASL signal and variance.

Figure 6 summarizes the 2D versus 3D comparison in all subjects and confirms the qualitative conclusions drawn from Fig. 5. These numbers were obtained by averaging the ASL signal and temporal standard deviation over the central 10 slices, excluding the ventricles. On average, 3D acquisition with $15^{\circ}$ flip-angle results in an increase of the tSNR by a factor of nearly 2 relative to $2 \mathrm{D}$ multislice. This exceeds the lower "limit" of 1.01 calculated from Eq. [1] for $N=24$ and $\alpha=15^{\circ}$. As noted above, the variance is not strongly influenced by flip-angle.

\section{RF Phase Cycling}

Figure 7 shows representative ASL images in one subject. The mean signal and variance are shown for the three different RF phase cycling schemes: constant, random, and RF-spoiled. There are several things to notice: first, all sequences produce similar signal intensity. Second, the variance of the RF-spoiled ASL signal is significantly lower than for constant phase cycling, as expected from steady-state signal theory. Signal variance is suppressed most dramatically in CSF (almost 4-fold), but also in the brain as a whole. This is expected, as CSF movement is large compared to brain tissue, in which motion is predominantly cardiac and respiratory (29). Third, random RF phase cycling produces slightly higher variance than RFspoiling ( $117^{\circ}$ phase increment). This is also predicted from steady-state theory, as the spoiling should be suboptimal for random RF phase cycling (24).

Figure 8 summarizes the results for all subjects. The figure shows measurements averaged over the 10 central slices, excluding the ventricles. On average, RF-spoiling 

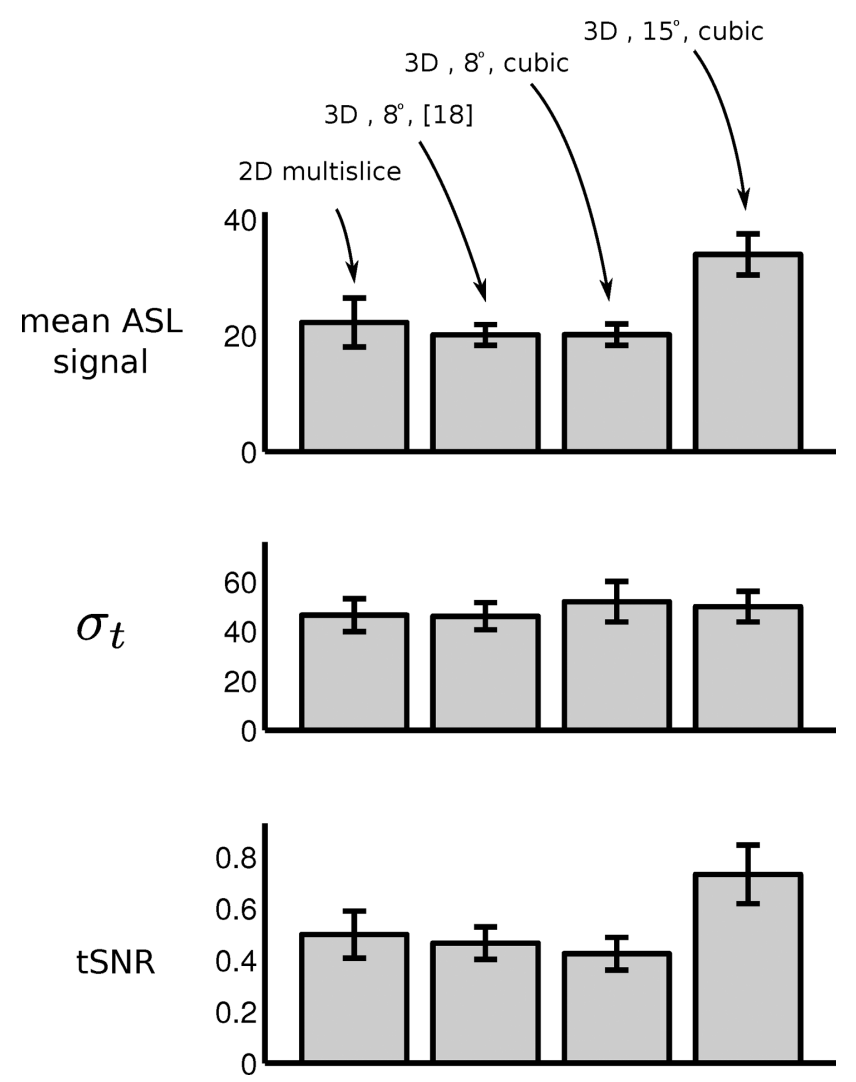

FIG. 6. Comparison of 2D multislice and segmented 3D spiral ASL, summary of results for subjects 1-5. Mean ASL signal, temporal standard deviation $\sigma_{\mathrm{t}}$, and tSNR are shown. 3D imaging with $15^{\circ}$ flip-angle produces a nearly 2 -fold increase in tSNR compared to 2D. The signal variance is not affected significantly by increasing the flip-angle from $8^{\circ}$ to $15^{\circ}$.

achieves a tSNR increase of $60 \%$ compared to the constantphase acquisitions.

\section{Functional MRI}

Figure 9 shows a representative activation map due to the visual stimulation paradigm obtained with segmented 3D spiral ASL. Robust activation can be seen in the visual cortex, where $Z$ scores exceed 10. A time course from a $3 \times 3$ pixel region-of-interest in the visual cortex shows a very clear activation pattern in the ASL signal. The mean contrast-to-noise ratio (CNR) in a $3 \times 3$ region-of-interest in the visual cortex was $9.1,9.2$, and 6.8 in subjects 6,7 , and 8 , respectively (Table 3 ).

\section{DISCUSSION}

Our measurements of tSNR in 2D multislice and 3D imaging with different RF phase cycling schemes show that the ASL signal behaves - at least qualitatively — according to steady-state signal theory. In particular, we have observed that (i) the temporal variance in 3D ASL is greater than in 2D multislice and (ii) random RF phase cycling is less optimal than RF-spoiling. The fact that steady-state MRI theory can be used to predict the behavior of transient subtractive ASL imaging is a powerful observation.
In conventional steady-state imaging sequences, RFspoiling is implemented with $117^{\circ}$ linear phase increment from shot-to-shot. It is possible that other values may be more optimal for subtractive transient imaging sequences such as the one used here. In fact, simulation results indicate that a value of $84^{\circ}$ may improve transverse signal spoiling and produce more "pure" $T_{1}$-weighting in transient imaging (30). To our knowledge, the optimal phase increment in subtractive transient RF-spoiled imaging has not been investigated and is a topic for future study.

Our theoretical calculations indicate that cubic flip-angle scheduling produces only modest overall k-space filtering (blurring in difference image space), while allowing significantly higher flip-angle and hence SNR compared to a schedule that produces constant signal in the individual tag/control images (18). Our in vivo observations confirm this prediction. In particular, we observed little appreciable difference regarding blurring between the cubic schedule and the flip-angle schedule in Ref. 18 (coronal views in Fig. 5). An additional advantage of a cubic schedule is that it is easy to implement on the MRI scanner and can be calculated for arbitrary $N$ and desired starting angle $\alpha(1)$.

Smith et al. (31) have developed a numerical optimization routine for designing a variable flip-angle schedule
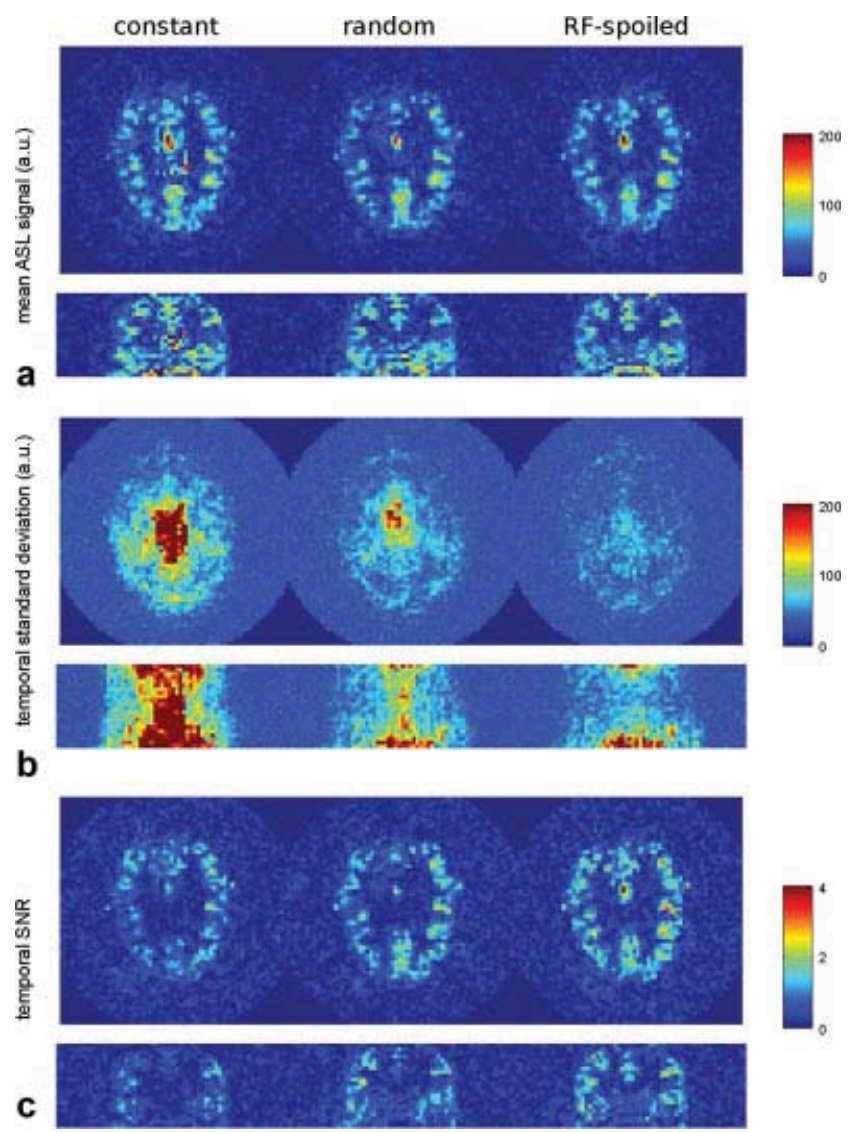

FIG. 7. Effect of RF phase cycling scheme on perfusion signal, timevariance, and tSNR in segmented 3D spiral ASL (subject 1). Results for three different RF phase cycling schemes are shown. RF-spoiling (right) greatly suppresses temporal variance, compared to constant (left) or random (middle) phase cycling. [Color figure can be viewed in the online issue, which is available at wileyonlinelibrary.com.] 

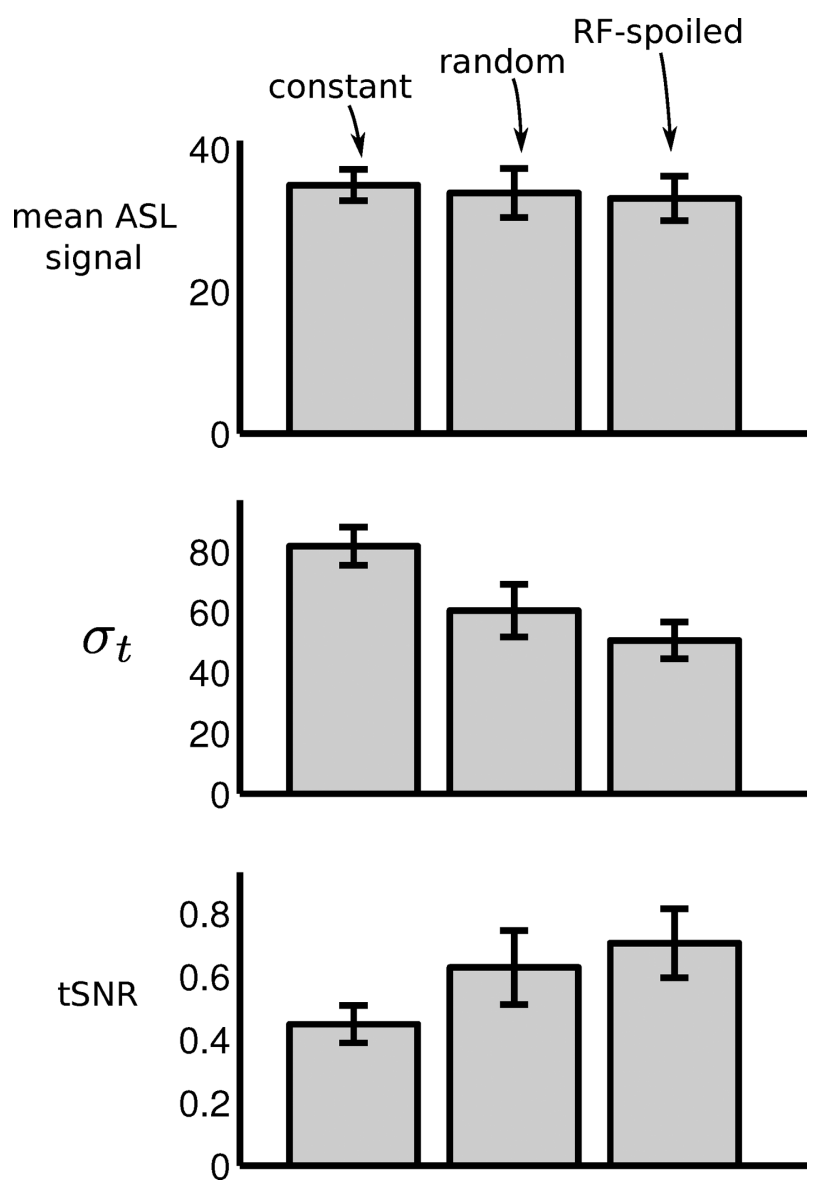

FIG. 8. Effect of RF phase cycling scheme on tSNR of the ASL signal, summary of results for subjects 1-5. Results are shown for both gradient-spoiled (constant) imaging, random phase cycling, and RFspoiling. On average, RF-spoiling improves tSNR by $60 \%$ compared to gradient-spoiling (constant RF phase). for subtractive balanced steady-state free precession imaging with linear phase-encode ordering, and it is possible that their general approach may be adapted to our application. The method in Ref. 31 defines three different cost functions, based on either (1) maximizing the area under the difference signal curve (such as the curves shown in Fig. 3), (2) maximizing the smallest difference signal, or (3) minimizing the blurring in the difference image. A heuristic multistep optimization routine is then performed with the goal of finding a global maximizer for one of the cost functions. In our application, we use center-out $\mathrm{kz}$-space ordering, and the SNR is therefore primarily determined by the initial flip-angle, and not the choice of flip-angle schedule. However, for a given initial flip-angle, the choice of flip-angle schedule influences the degree of blurring in the ASL difference image. It is possible that the method in Ref. 31 can be used with a modified cost function that balances the choice of initial flip-angle against the degree of image blurring, and that the resulting schedule can produce a more favorable tradeoff between SNR and blur than the simple polynomial schedules proposed here (at the cost of increased complexity).

Finally, we note that RF-spoiling may be particularly advantageous when analyzing complex (instead of magnitude) image data (14). Image phase contains information about blood flow and hence perfusion, due to the flowencoding effect of the slice-select and imaging gradients. In the absence of RF-spoiling, spin phase depends on not only velocity-encoding but also on spin history, and it is not clear whether the phase-signal in non-RF-spoiled ASL reflects tissue perfusion.

\section{CONCLUSIONS}

We have shown that the tSNR of the dynamic ASL signal can be increased 2-fold by using a segmented low-flip-angle $3 \mathrm{D}$ readout, and that segmented 3D spiral ASL should be implemented using RF-spoiling. Our results also suggest

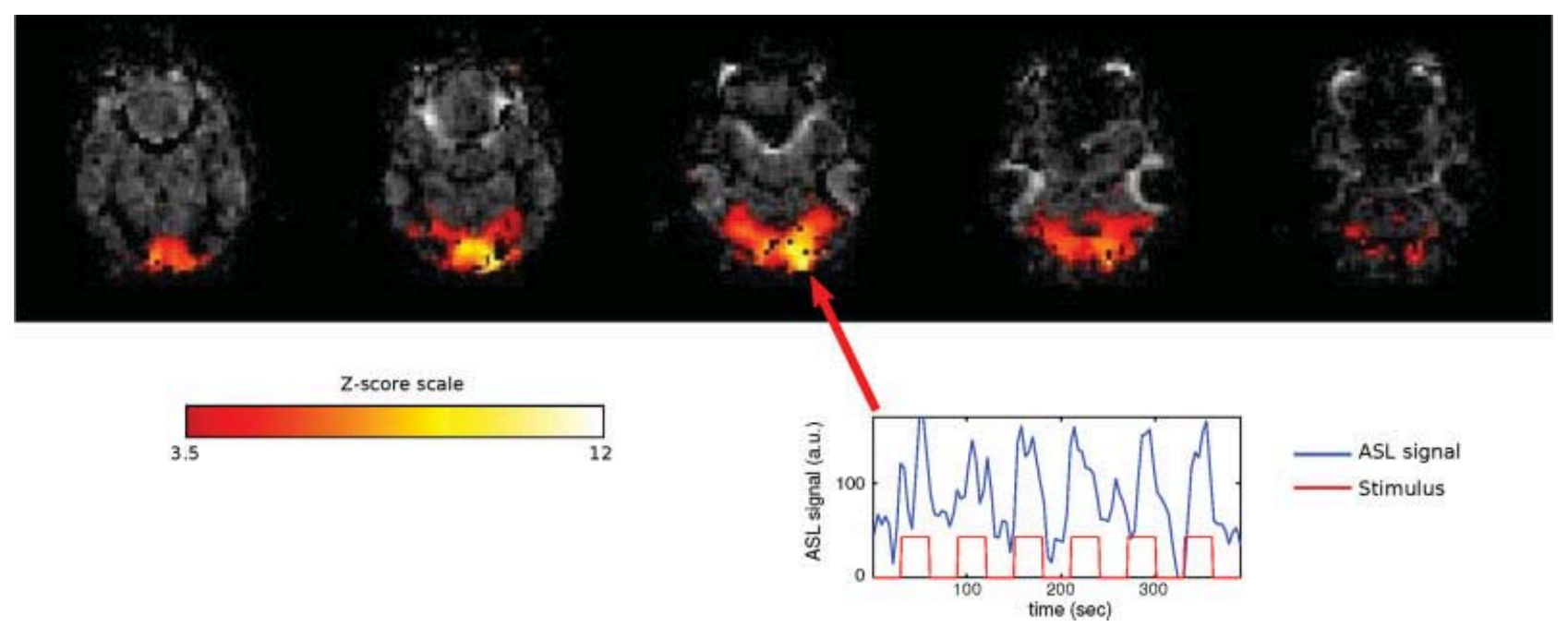

FIG. 9. Functional imaging using segmented 3D spiral ASL, visual checkerboard stimulation (subject 6). Z-score thresholded at 3.5, overlaid onto the mean ASL (subtraction) image. The ASL time-course signal and functional stimulus timing are also shown, for a voxel pointed to by the read arrow. [Color figure can be viewed in the online issue, which is available at wileyonlinelibrary.com.] 
Table 3

Summary of functional imaging results using segmented 3D spiral ASL, visual checkerboard stimulation (subjects 6, 7, and 8).

\begin{tabular}{lcccc}
\hline Subject & $\alpha$ schedule & $\begin{array}{c}\text { Starting } \\
\text { flip-angle }(\alpha(1))\end{array}$ & $\begin{array}{c}\text { RF phase } \\
\text { cycling }\end{array}$ & CNR \\
\hline 6 & cubic & $15^{\circ}$ & RF-spoiled & 9.1 \\
7 & cubic & $15^{\circ}$ & RF-spoiled & 9.2 \\
8 & cubic & $15^{\circ}$ & RF-spoiled & 6.8 \\
\hline
\end{tabular}

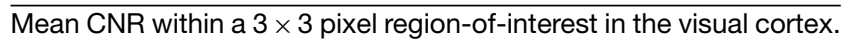

that a cubic flip-angle schedule, which is simple to implement, produces only modest blurring in the ASL difference images and that the tSNR in segmented 3D spiral ASL scales with imaging flip-angle. Our measurements indicate that steady-state MRI sequence theory can be used to predict the qualitative behavior of transient subtractive ASL imaging, such as the effect of different RF phase cycling schemes.

\section{REFERENCES}

1. Detre JA, Leigh JS, Williams DS, Koretsky AP. Perfusion imaging. Magn Reson Med 1992;23:37-45.

2. Golay X, Hendrikse J, Lim TC. Perfusion imaging using arterial spin labeling. Top Magn Reson Imaging 2004;15:10-27.

3. Jiang L, Kim M, Chodkowski B, Donahue MJ, Pekar JJ, Zijl PC, Albert M. Reliability and reproducibility of perfusion MRI in cognitively normal subjects. Magn Reson Imaging 2010;28:1283-1289.

4. Petersen ET, Mouridsen K, Golay X. The quasar reproducibility study, part ii: results from a multi-center arterial spin labeling test-retest study. Neuroimage 2010;49:104-113.

5. Wang Y, Saykin AJ, Pfeuffer J, Lin C, Mosier KM, Shen L, Kim S, Hutchins GD. Regional reproducibility of pulsed arterial spin labeling perfusion imaging at 3T. Neuroimage 2011;54:1188-1195.

6. Aguirre GK, Zarahn E, D'esposito M. The variability of human, BOLD hemodynamic responses. Neuroimage 1998;8:360-369.

7. Deibler AR, Pollock JM, Kraft RA, Tan H, Burdette JH, Maldjian JA. Arterial spin-labeling in routine clinical practice, part 1: technique and artifacts. Am J Neuroradiol 2008;29:1228-1234.

8. Deibler AR, Pollock JM, Kraft RA, Tan H, Burdette JH, Maldjian JA. Arterial spin-labeling in routine clinical practice, part 2: hypoperfusion patterns. Am J Neuroradiol 2008;29:1235-1241.

9. Deibler AR, Pollock JM, Kraft RA, Tan H, Burdette JH, Maldjian JA. Arterial spin-labeling in routine clinical practice, part 3: hyperperfusion patterns. Am J Neuroradiol 2008;29:1428-1435.

10. Wang Z, Fernández-Seara M, Alsop DC, Liu WC, Flax JF, Benasich AA, Detre JA. Assessment of functional development in normal infant brain using arterial spin labeled perfusion MRI. Neuroimage 2008;39: 973-978.

11. Detre JA, Wang J, Wang Z, Rao H. Arterial spin-labeled perfusion MRI in basic and clinical neuroscience. Curr Opin Neurol 2009;22:348-355.

12. Perthen JE, Bydder M, Restom K, Liu TT. SNR and functional sensitivity of BOLD and perfusion-based fMRI using arterial spin labeling with spiral SENSE at $3 \mathrm{~T}$. Magn Reson Imaging 2008;26:513-522.
13. Yang Y, Gu H, Stein EA. Simultaneous MRI acquisition of blood volume, blood flow, and blood oxygenation information during brain activation. Magn Reson Med 2004;52:1407-1417.

14. Hernandez-Garcia L, Vazquez AL, Rowe DB. Complex-valued analysis of arterial spin labeling-based functional magnetic resonance imaging signals. Magn Reson Med 2009;62:1597-1608.

15. Günther M, Oshio K, Feinberg DA. Single-shot 3D imaging techniques improve arterial spin labeling perfusion measurements. Magn Reson Med 2005;54:491-498.

16. Talagala SL, Ye FQ, Ledden PJ, Chesnick S. Whole-brain 3D perfusion MRI at 3.0 T using CASL with a separate labeling coil. Magn Reson Med 2004;52:131-140.

17. Talagala SL, Slavin GS, Ostuni J, Chesnick S. CASL perfusion MRI with non-segmented low flip angle 3D EPI. In Proceedings of the 14th Annual Meeting of ISMRM, Seattle, Washington, USA, 2006. p. 3422.

18. Gai ND, Talagala SL, Butman JA. Whole-brain cerebral blood flow mapping using 3D echo planar imaging and pulsed arterial tagging. J Magn Reson Imaging 2011;33:287-295.

19. Fernández-Seara MA, Wang Z, Wang J, Rao HY, Guenther M, Feinberg DA, Detre JA. Continuous arterial spin labeling perfusion measurements using single shot 3D GRASE at 3 T. Magn Reson Med 2005;54:12411247.

20. Duhamel G, Alsop DC. Single-shot susceptibility insensitive whole brain 3D fMRI with ASL. In Proceedings of the 12th Annual Meeting of ISMRM, Kyoto, Japan, 2004. p. 518.

21. Dai W, Garcia D, Bazelaire C, Alsop DC. Continuous flow-driven inversion for arterial spin labeling using pulsed radio frequency and gradient fields. Magn Reson Med 2008;60:1488-1497.

22. $\mathrm{Hu} \mathrm{Y}$, Glover GH. Three-dimensional spiral technique for highresolution functional MRI. Magn Reson Med 2007;58:947-951.

23. Scheffler K. A pictorial description of steady-states in rapid magnetic resonance imaging. Concepts Magn Reson 1999;11:291-304.

24. Zur Y, Wood ML, Neuringer LJ. Spoiling of transverse magnetization in steady-state sequences. Magn Reson Med 1991;21:251-263.

25. Alsop DC, Detre JA. Reduced transit-time sensitivity in noninvasive magnetic resonance imaging of human cerebral blood flow. J Cereb Blood Flow Metab 1996;16:1236-1249.

26. Wang J, Alsop DC, Li L, Listerud J, Gonzalez-At JB, Schnall MD, Detre JA. Comparison of quantitative perfusion imaging using arterial spin labeling at 1.5 and 4.0 Tesla. Magn Reson Med 2002;48:242254.

27. Jahanian H, Noll DC, Hernandez-Garcia L. Bo field inhomogeneity considerations in pseudo-continuous arterial spin labeling (pCASL): effects on tagging efficiency and correction strategy. NMR Biomed, 2011;24:1202-1209.

28. Press WH, Flannery BP, Teukolsky SA, Vetterling WT. Numerical recipes in C: the art of scientific computing. New York: Cambridge University Press; 1992.

29. Raj D, Anderson AW, Gore JC. Respiratory effects in human functional magnetic resonance imaging due to bulk susceptibility changes. Phys Med Biol 2001;46:3331-3340.

30. Epstein FH, Brookeman JR. Spoiling of transverse magnetization in gradient-echo (GRE) imaging during the approach to steady state. Magn Reson Med 1996;35:237-245.

31. Smith T, Zun Z, Wong EC, Nayak KS. Design and use of variable flip angle schedules in transient balanced SSFP subtractive imaging. Magn Reson Med 2010;63:537-542. 